\title{
Adaptação transcultural da Healthcare Failure Mode and Effect Analisys (HFMEA) para o uso no Brasil: nota prévia
}

Transcultural adaptation of the Healthcare Failure Mode and Effect Analysis (HFMEA) for use em Brazil: preview

Adaptacion transcultural del Healthcare Failure Mode and Effect Analysis (HFMEA) para su uso en Brasil: nota anterior

Thiana Sebben Pasa

ORCID: https://orcid.org/0000-0003-3931-4149 Pontifícia Universidade Católica do Rio Grande do Sul, Brasil E-mail: thianasp@ @otmail.combr

Tania Solange Bosi de Souza Magnago ORCID: https://orcid.org/0000-0002-5308-1604 Universidade Federal de Santa Maria, Brasil E-mail: magnago.tania@gmail.com Janete de Souza Urbanetto ORCID: https://orcid.org/0000-0002-4697-1641 Pontifícia Universidade Católica do Rio Grande do Sul, Brasil E-mail: jurbanetto@pucrs.br

\begin{abstract}
Resumo
Objetivo: apresentar o planejamento da adaptação transcultural da Healthcare Failure Mode and Effect Analysis (HFMEA) para a cultura brasileira. Método: trata-se de um estudo metodológico que utilizará o processo de adaptação transcultural proposto por Beaton e colaboradores, que compreende seis etapas: tradução inicial, síntese das traduções, retrotraduções, comitê de especialistas, teste da versão pré-finalizada e avaliação e revisão de documentos produzidos. Para compor o comitê de especialistas serão priorizados profissionais de diferentes regiões do país, a fim de abranger as diferenças culturais e evitar vieses de linguagem. O teste da versão pré-finalizada será realizado com membros do Núcleo de Segurança do Paciente de um hospital do sul do Brasil, mediante a análise de um caso proposto pelas pesquisadoras. Serão respeitados os preceitos éticos propostos pela Resolução no 466/2012 do Conselho Nacional de Saúde. Considerações finais: espera-se que a adaptação transcultural do HFMEA para a cultura brasileira impulsione os gestores e as instituições para realizarem a análise de riscos de forma sistemática. O método poderá auxiliar na identificação de vulnerabilidades dos processos, repercutindo diretamente na assistência prestada aos pacientes e no estabelecimento de práticas seguras.

Palavras-chave: Segurança do paciente; Análise do modo e do efeito de falhas na assistência à saúde; Tradução; Gestão de riscos.

Abstract

Objective: to present the planning of the cross-cultural adaptation of the HealthCare Failure Mode and Effect Analysis (HFMEA) to the Brazilian culture. Method: this is a methodological study that will use the process of cross-cultural adaptation proposed by Beaton and collaborators, which includes six stages: initial translation, synthesis of translations, back-translations, expert committee, assessment of the pre-final version and evaluation and review of produced documents. To make up the committee of experts, professionals from different regions of the country will be selected to reach cultural differences and avoid language bias. The assessment of the pre-final version will be carried out with members of the Patient Safety Center of a hospital in southern Brazil, through the analysis of a case proposed by the researchers. The ethical precepts proposed by Resolution no. 466/2012 of the National Health Council will be respected. Final considerations: it is expected that the crosscultural adaptation of HFMEA to Brazilian culture will encourage managers and institutions to carry out risk analysis in a systematic way. The method can assist in the identification of vulnerabilities in the process, directly affecting the care provided to patients and the establishment of safe practices.
\end{abstract}

Keywords: Patient safety; Healthcare failure mode and effect analysis; Translating; Risk management.

\section{Resumen}

Objetivo: Presentar la planificación de la adaptación intercultural del Healthcare Failure Mode and Effect Analysis (HFMEA) (HFMEA) para la cultura brasileña. Método: Este es un estudio metodológico que utilizará el proceso de adaptación intercultural propuesto por Beaton et al., que comprende seis etapas: traducción inicial, síntesis de traducciones, traducciones posteriores, comité de expertos, pruebas de versiones pre-finalizadas y evaluación y revisión de documentos producidos. Para 
conformar el comité de expertos se priorizarán profesionales de diferentes regiones del país, con el fin de cubrir las diferencias culturales y evitar las opiniones lingüísticas. La prueba de la versión pre-acabada se realizará con miembros del Centro de Seguridad del Paciente de un hospital en el sur de Brasil, a través del análisis de un caso propuesto por los investigadores. Se respetarán los preceptos éticos propuestos por la Resolución N. ${ }^{\circ}$ 466/2012del Consejo Nacional de Salud. Notas finales: se espera que la adaptación intercultural de HFMEA a la cultura brasileña impulse a los gerentes e instituciones a realizar análisis de riesgos sistemáticamente. El método puede ayudar a identificar vulnerabilidades en los procesos, reflexionando directamente sobre la atención brindada a los pacientes y en el establecimiento de prácticas seguras.

Palabras clave: Seguridad del paciente; Análisis de modo y efecto de fallas en la atención de la salud; Traducción; Gestión de riesgos.

\section{Introdução}

A necessidade de atendimento em instituições de saúde não é isenta de riscos, uma vez que estes locais possuem processos complexos e fragilidades organizacionais e assistenciais, que podem propiciar a ocorrência de incidentes e até mesmo de eventos adversos (Sagawa et al., 2019). Define-se incidente como o evento ou circunstância que poderia causar, ou que causou danos desnecessários ao paciente, durante a assistência em saúde. Já o evento adverso é o incidente que causou danos (Runcinan et al., 2009; Agência Nacional de Vigilância Sanitária, 2013; Ministério da Saúde A, 2013).

Estima-se que, anualmente, ocorram cerca de 134 milhões de eventos adversos decorrentes de cuidados de saúde inseguros e que 2.620.412 destes tenham o óbito como desfecho (Institute of Medicine Committee on Quality of Health Care in America, 2018). Estudo realizado no Brasil identificou prevalência de 33,8 incidentes notificados para cada 1.000 internações e que o percentual de notificações teve aumento gradualmente nos três últimos anos de coleta (Figueiredo, Oliveira e Silva, Brito, D’Innocenzo, 2018). Corroboram com este dado, as 330.536 notificações de incidentes ocorridos entre os anos de 2014 e 2019 no Brasil e notificados no sistema da Agência Nacional de Vigilância Sanitária (ANVISA) (Silva et al., 2020).

Notificar os incidentes auxilia os gestores na identificação da causa que levou a sua ocorrência, pois a descrição das circunstâncias em que o evento ocorreu, viabiliza uma análise do processo envolvido e suas possíveis falhas. Para Derosier, Stalhandske, Bagian, Nudell (2002), os relatórios provenientes das notificações se concentram em analisar os incidentes após a sua ocorrência; entretanto, há métodos que realizam a análise prospectiva das vulnerabilidades de um processo, ou seja, métodos que identificam falhas de processos antes que estes sejam implantados. Utilizar-se de métodos de análise de risco torna-se um aliado na prevenção de incidentes de segurança (Silva, Cassiani, 2013)

Com a finalidade de disponibilizar um método para análise prospectiva de riscos voltados para processos da área da saúde, mais especificamente da área da segurança do paciente, em 2002, pesquisadores desenvolveram o HFMEA (Healthcare Failure Mode and Effect Analysis) (Derosier et al., 2002). Este é uma adaptação de um método utilizado na área da engenharia, o Modo de Falha e Análise do Efeito (FMEA), que tem por finalidade a análise de processos e produtos.

O HFMEA é composto por cinco etapas e utiliza uma abordagem interdisciplinar. Tem por objetivos avaliar prospectivamente processos de assistência em saúde e apontar que para cada modo de falha, há um possível efeito e a gravidade deste deve ser identificada. A partir da identificação de processos com maior gravidade, por meio dos critérios estabelecidos no método, são elencados as etapas prioritárias que necessitam de planejamento e proposição de ações de prevenção (Derosier et al., 2002).

Quando comparada a utilização do FMEA versus HFMEA para analisar riscos em setor da área da saúde, identificouse que há diferenças entre os dois métodos e apontou o HFMEA como mais simples e adequado para a aplicação nesta área, além de ressaltar a efetividade da Matriz de Pontuação de Risco e da Árvore de Decisão utilizadas neste método (Rah, Manger, Yock, Kim, 2016). Ainda, a utilização do método é um importante aliado para justificar as possíveis alterações que devem ocorrer, relacionados ao processo de trabalho (Overdevest, Hees, Lagerburg, Kloeze, Straten, 2014).

Observa-se que a realização de uma avaliação de risco prospectiva auxilia os profissionais e gestores na identificação de potenciais fragilidades dos processos e no planejamento e proposição de ações factíveis na realidade da instituição. No 
Brasil, o HFMEA vem sendo utilizado, porém observa-se o emprego da tradução livre do método (Salvador, 2018; Sousa, 2014). Entretanto, a utilização de tradução livre de instrumento ou método construído em outro idioma e consequentemente outra cultura, pode propiciar equívocos no entendimento do processo e de conceitos.

A partir do exposto, tem-se por objetivo apresentar o planejamento da adaptação transcultural da HealthCare Failure Mode and Effect Analysis (HFMEA) para a cultura brasileira.

\section{Metodologia}

Trata-se de uma proposta de pesquisa apresentada ao Programa de Pós-Graduação em Gerontologia Biomédica, Nível - Doutorado, da Pontifícia Universidade Católica do Rio Grande do Sul (PUC-RS). Realizar-se-á um estudo metodológico, com vistas a adaptação transcultural de um método de análise de risco, a HFMEA, para a língua portuguesa e cultura do Brasil. Estudos dessa natureza possibilitam a avaliação e validação de instrumentos e métodos de pesquisas, verificando sua confiabilidade e aplicação por outros pesquisadores (Polit, Beck, 2019).

Para o início do processo de adaptação transcultural do método HFMEA para a cultura brasileira, obteve-se a permissão, via correio eletrônico, de Joseph DeRosier, professor da Universidade de Michigan, Estados Unidos da América (EUA), um dos autores responsáveis pela adaptação do método para a área da saúde.

A HFMEA é um método adaptado da área da engenharia para a área da saúde, com o objetivo de realizar a análise de processos relacionados, principalmente, à segurança do paciente. O método é composto por cinco etapas, conforme figura abaixo:

Figura 1: Fases do método HFMEA - Adaptado (Derosier, 2018).

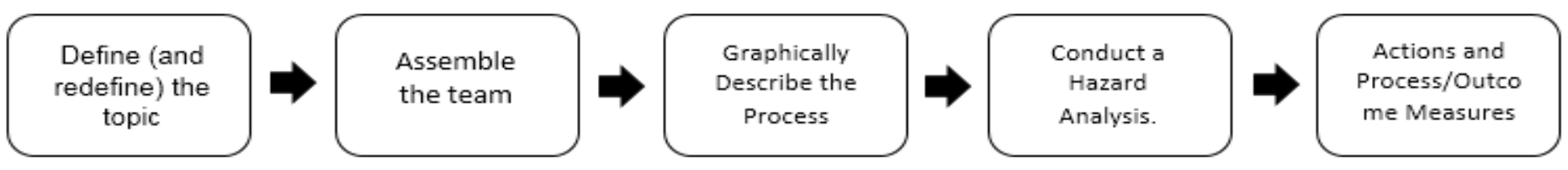

Fonte: Autores.

Para entendimento do método, foi realizada a livre tradução das etapas, sendo, Step 1 - Define the topic [definição do processo]; Step 2 - Assemble the team [constituição da equipe]; Step 3 - Graphically describe the Process [descrição de fluxos do processo]; Step 4 - Conduct a Hazard Analysis [análise e identificação dos modos de falhas e seus efeitos na assistência ao paciente]; Step 5 - Actions and Process/Outcome measures [planejamento de ações para os processos apontados como críticos na etapa anterior] (Derosier, 2018). Ressalta-se que no decorrer do processo de adaptação transcultural, estas traduções poderão ser alteradas.

Para realizar o processo de adaptação transcultural (ACT), foi utilizado o modelo proposto por Beaton e colaboradores, o qual preconiza a realização de seis etapas (Beaton, Bombardier, Guillemin, Ferraz, 2007):

- Etapa 1: Tradução inicial: realizada por dois tradutores com fluência no inglês (americano) e com o português do Brasil como língua materna. Essas traduções serão realizadas de forma independente, e produzirão as versões T1 e T2. Um dos tradutores conhecerá os objetivos da tradução e o outro não.

- Etapa II - Síntese das traduções: as traduções T1 e T2 serão submetidas a análise de um pequeno comitê, composto pelos pesquisadores principais, os tradutores e um profissional com domínio do FMEA. Este comitê tem a finalidade de diminuir as incongruências decorrentes das traduções e colaborar com a elaboração da versão final da síntese de T-12. 
- Etapa III - Back Translation ou Retrotradução: tradução da versão síntese (T-12) da língua portuguesa para o inglês, que originará as versões B1 e B2. Para isso, dois tradutores da área da saúde, com o inglês como língua materna e que não participaram de etapas anteriores, realizarão as traduções de forma independente. Ainda nesta etapa, será inserido um terceiro tradutor que irá compilar as retrotraduções, considerando a versão original e as alterações culturais necessárias. Novamente um pequeno comitê será composto para a elaboração da versão final de B-12.

- Etapa IV - Comitê de especialistas: será constituído um comitê, composto por até cinco profissionais experts na temática Segurança do Paciente e que tenham conhecimento do FMEA ou HFMEA. Serão priorizados profissionais de diferentes regiões do país, a fim de abranger as diferenças culturais e evitar vieses de linguagem que possam interferir na interpretação do método. A finalidade deste Comitê é elaborar a versão pré-finalizada do HFMEA versão brasileira, para testes de campo. Nesta etapa ocorrerá a validação de conteúdo por meio da análise do Índice de Validade de Conteúdo (IVC) quanto a concordância nas melhores pontuações de equivalências semântica, idiomática, conceitual e cultural da versão proposta da HFMEA para o uso no Brasil.

- Etapa V - Teste da versão pré-finalizada: teste piloto, que será realizado com os membros do Núcleo de Segurança do Paciente de um hospital universitário do Sul do país. Para isso, serão formados dois grupos de profissionais que irão utilizar o HFMEA adaptado transculturalmente para o português do Brasil e aplicá-lo na análise do processo de avaliação do risco de quedas dos pacientes adultos internados, conforme estudo de caso proposto pelas pesquisadoras. Cada grupo irá avaliar o mesmo caso de forma independente. Ao término da avaliação, será avaliado e comparado a compreensão dos profissionais na aplicação do método e a praticabilidade deste, por meio de um questionário com questões objetivas que compreendem entendimento das etapas, facilidade de seguir as etapas e facilidade no preenchimento no decorrer de sua execução (Coluci, Alexandre, 2009). As respostas referentes a este questionário serão do tipo Likert e a análise será calculada a partir da frequência absoluta (n) e relativa (\%) das variáveis e respostas ao questionário Likert. Também, serão realizadas comparações entre os resultados obtidos no IVC (Comitê de especialistas) e a praticabilidade (Integrantes do NSP), por meio do Teste de Wilcoxon, com nível de significância de 5\%.

- Etapa VI - Avaliação e revisão de todos os documentos produzidos: nesta etapa todos documentos produzidos até o momento serão conferidos e analisados criticamente pelos pesquisadores responsáveis. A versão final do HFMEA adaptado para cultura brasileira será encaminhada ao autor da estrutura original, para a aprovação da versão adaptada.

A utilização do método proposto por Beaton e colaboradores (2007), para a ACT, é destacada na área da enfermagem por sua importância no que tange ao consenso em relação ao referencial teórico-metodólogico adequado, sendo referência internacional para estudos desta natureza (Machado, Fernandes, Soares, Gouveia, Silva, 2018). A realização desse processo, de forma sistematizada, conforme proposto por Beaton e colaboradores (2007), irá auxiliar no desenvolvimento de um instrumento equivalente ao original, uma vez que avalia a equivalência em diferentes aspectos (semântica, idiomática, conceitual e cultural). Apesar desse processo demandar tempo e ser dispendioso, permitirá a utilização de ferramentas s em diferentes países ou em um mesmo país, com culturas diferentes, evitando possíveis vieses (Dortas Júnior, Lupi, Dias, Guimarães, Valle, 2016).

Para o desenvolvimento do estudo, serão seguidos os aspectos éticos estabelecidos pelas Diretrizes e Normas Regulamentadoras de Pesquisa Envolvendo seres Humanos, estabelecidas na Resolução nº66/2012 do Conselho Nacional de Saúde/Ministério da Saúde (Ministério da Saúde, 2013). Aos participantes, será disponibilizado o Termo de Consentimento Livre e Esclarecido (TCLE), para assinatura mediante o aceite em participar. A pesquisa foi submetida e aprovada pela Comissão Científica da Pontifícia Universidade Católica do Rio Grande do Sul - PUC/RS e pelo Comitê de Ética em Pesquisa (CEP) da instituição em que será desenvolvida pesquisa, com aprovação sob parecer № 29578220.0.0000.5346. 


\section{Considerações Finais}

A partir deste estudo, pretende-se realizar a adaptação transcultural da HFMEA. Espera-se que o desenvolvimento desta pesquisa contribua na promoção da segurança do paciente em âmbito nacional, uma vez que a adaptação transcultural da HFMEA, para a língua portuguesa e sua posterior validação, irão subsidiar os gestores e as instituições para realizarem a análise de riscos de forma sistemática. Este método poderá auxiliar na identificação de vulnerabilidades dos processos, antes que elas atinjam os pacientes, repercutindo diretamente na assistência prestada aos mesmos.

Após o processo de adaptação transcultural, sugere-se que estudos utilizando a HFMEA sejam realizados, avaliando diferentes processos relacionados a assistência a saúde. A validação deste método em diferentes processos assistenciais poderá subsidiar o planejamento de ações, protocolos e serviços, com vistas a eliminar os riscos antes destes produtos serem colocados em prática.

\section{Agradecimentos}

O presente trabalho foi realizado com apoio da Coordenação de Aperfeiçoamento de Pessoal Nivel Superior - Brasil (CAPES) - Código de Financiamento 001.

\section{Referências}

Agência Nacional de Vigilância Sanitária (2013). Resolução RDC No 36, DE 25 DE JULHO DE 2013. Institui ações para a segurança do paciente em serviços de saúde e dá outras providências. Ministério da Saúde. Diário Oficiao [da] República Federativa do Brasil, Brasilia - DF.

Beaton, D., Bombardier, C., Guillemin, F., \& Ferraz, M. B. (2007). Recommendations for the Cross-Cultural Adaptation of the DASH \& QuickDASH Outcome Measures. Institute for Work \& Health. Toronto.

Coluci, M. Z. O., \& Alexandre, N. M. C. (2009). Development of a Questionnaire to Evoluate the Usability of Assessment Instruments. Revista Enfermagem UERJ, 17(3), 378-382.

Derosier, J. M. (2018). Healthcare Failure Mode Effect Analysis (HFMEA) Process. Center for Healthcare Engineering and Patient Safety. University of Michigan.

Derosier, J., Stalhandske, E., Bagian, J. P., \& Nudell, T. (2002). Using Health Care Failure Mode and Effect Analysis ${ }^{\mathrm{TM}}$ : The VA National Center for Patient Safety's Prospective Risk Analysis System. The Joint Commission Journal on Quality Improvement, 28(5), $248-267$.

Dortas Junior, S. D., Lupi, O., Dias, G. A. C., Guimaraes, M. B. S., \& Valle, S. O. R. (2016). Adaptação Transcultural e validação de questionários na área da saúde. Brazilian Journal of Allergy and Immunology, 4(1), 26-30.

Figueiredo, M. L., Oliveira e Silva, C. S., Brito, M. F. S. F., D’Innocenzo M. (2018). Analysis of incidents notified in a general hospital. Revista Brasileira de Enfermagem, 71(1), 111-119.

Institute of Medicine Committee on Quality of Health Care in America. (2018). Crossing the global quality chasm: Improving health care worldwide.: The National Academies Press.

Machado, R. S., Fernandes, A. D. B. F., Oliveira, A. L. C. B., Soares, L. S., Gouveia, M. T. O., \& Silva, G. R. F. (2018). Cross-cultural adaptation methods of instruments in the nursing area. Revista Gaúcha de Enfermagem. 39(e2017), 1-11.

Ministério da Saúde (Brasil) A. (2013). Portaria nº 529, de $1^{\circ}$ de abril de 2013. Institui o Programa Nacional de Segurança do Paciente (PNSP). Diário Oficiao [da] República Federativa do Brasil, Brasilia - DF.

Ministério da Saúde (2013). Resolução nº 466/12 do Conselho Nacional de Saúde. Ministério da Saúde. Brasília-DF.

Overdevest E. P., Hees, J. W. H van., Lagerburg, V., Kloeze, C., Straten, A. H. M van. (2014). Healthcare failure mode effect analysis of a miniaturized extracorporeal bypass circuit. Perfusion, 29(4), 301-306.

Polit, D. F., \& Beck, C. T. (2019). Fundamentos de pesquisa em enfermagem: avaliação de evidências para a prática da enfermagem. (9a ed.), Artmed.

Rah, J. E., Manger, R. P., Yock, A. D., \& Kim, G. Y. (2016). A comparison of two prospective risk analysis methods: Traditional FMEA and a modified healthcare FMEA. Medical Physics, 43(12), 6347-6353.

Runcinan, W., Hibbert, P., Thomson, R., Schaaf, T. V. D., Sherman, H., \& Lewall, P. (2009). Towards an International Classification for Patient Safety: key concepts and terms. International Society for Quality in Health Care, 21, 18-26.

Sagawa, M. R., Silva, A. E. B. de C., Lima, J. C. de, Bezerra, A. L. Q., Costa, N. N., Sousa, M. R. G. de, \& Gimenez, F. R. E. (2019). Análise de circunstâncias notificáveis: incidentes que podem comprometer a segurança dos pacientes. Cogitare Enfermagem, 24, 1-12. 
Research, Society and Development, v. 10, n. 6, e15310615285, 2021

(CC BY 4.0) | ISSN 2525-3409 | DOI: http://dx.doi.org/10.33448/rsd-v10i6.15285

Salvador, C. (2018). Segurança do paciente e análise de risco na teleterapia conformacional em um centro de tratamento oncológico do sul do Brasil [dissertação]. Santa Catarina: Instituição Federal de Educação, Ciência e Tecnologia.

Silva, A. E. B. D. C., \& Cassiani, S. H. D. B. (2013). Análise prospectiva de risco do processo de administração de medicamentos anti-infecciosos. Revista Latino-Americana de Enfermagem, 21(número especial), 01-09.

Silva H. R., Costa, R. H. F., Neto, J. C. P., Macedo Júnior, C. A. A. de., do Nascimento, P. B., Moraes, R. dos A., \& Pires, Y. M. S. (2020). Analysis of incidents notified to the National Health Surveillance Notification System (NOTIVISA) in Brazil from 2014 to 2019. Research, Society and Development, 9(7), 2-17.

Sousa, M. C. A de. (2014). Aplicação da Ferramenta de Gerenciamento de Risco HFMEA no Setor de Expurgo do Centro de Material e Esterelização [dissertação]. São Paulo: Universidade Federal de Campinas. 